\title{
Legislación Colombiana frente a la evaluación escolar de la población con necesidades educativas no convencionales
}

Colombian Legislation against academic evaluation in children with nonconventional educational needs

\section{Volumen 20, Número 2 \\ Mayo - Agosto \\ pp. 1-18}

\section{Luz Nelly Ruiz González}

\section{Citar este documento según modelo APA}

Ruiz González, Luz Nelly. (2020). Legislación Colombiana frente a la evaluación escolar de la población con necesidades educativas no convencionales. Revista Actualidades Investigativas en Educación, 20(2), 1-18. Doi. 10.15517/aie.v20i2.41646 


\title{
Legislación Colombiana frente a la evaluación escolar de la población con necesidades educativas no convencionales \\ Colombian Legislation against academic evaluation in children with non-conventional educational needs
}

\section{Luz Nelly Ruiz González}

\begin{abstract}
Resumen: El presente ensayo tiene como objetivo exponer y analizar la situación que vive la población escolar franja media- que presenta características no convencionales de aprendizaje, es decir, características no ajustadas a la categorización de discapacidad ni de regularidad. En efecto, al establecer los estándares de evaluación, los países definen, mediante políticas de Estado expuestas en la legislación educativa, unas expectativas de aprendizaje basadas en un prospecto definido como regular; al otro lado de la balanza se encuentra la población definida como NEE (Necesidades Educativas Especiales) que presenta dificultades generadas por su discapacidad. Sin embargo, entre la población regular y la población con discapacidad, existe una población no reconocida en política pública, con una amplia gama de estilos y características de aprendizaje, gama a la que en el presente documento se le reconoce como franja media con características no convencionales de aprendizaje. Dada la zozobra que supone para una persona ser evaluada con un patrón de referencia distante de la posibilidad personal de respuesta, al exponer a esta población y analizar sus particulares circunstancias, de cara a las políticas públicas y a la cotidianidad escolar, se concluye que el Estado no ha podido particularizar el currículo para atender las condiciones singulares de aprendizaje de esta población. En consecuencia, se parte de la revisión de diferentes aportes de investigaciones relacionadas, con el fin de profundizar en el conocimiento de esta problemática y proponer algunos lineamientos aplicables a la metodología empleada para la adecuación del currículo, cuyo componente de evaluación sea consecuente y particularice las condiciones de la niñez objeto de este abordaje.
\end{abstract}

Palabras clave: legislación educativa, dificultad en el aprendizaje, evaluación de la educación, necesidades no convencionales.

Summary: This trial aims to expose and analyse the situation experienced by the school population - middle range - which has unconventional learning characteristics, i.e. characteristics not in line with the categorization of disability or regularity. Indeed, in setting assessment standards, countries define, through State policies set out in educational legislation, learning expectations based on a prospectus defined as regular; on the other side of the balance is the population defined as SEN (Special Educational Needs) which presents difficulties generated by their disability. However, among the regular population and the population with disabilities, there is a population not recognized in public policy, with a wide range of styles and learning characteristics, a range which in this document is recognized as a middle range with unconventional learning characteristics. Given the zozobra that means for a person to be evaluated with a reference pattern distant from the personal possibility of response, by exposing this population and analyzing its particular circumstances, in the face of public policies and school daily life, it is concluded that the State has not been able to particularize the curriculum to meet the unique learning conditions of this population. Consequently, it is based on the review of different contributions of related research, in order to deepen the knowledge of this problem and propose some guidelines applicable to the methodology used for the adequacy of the curriculum, whose evaluation component is consequential and to particularize the conditions of the children subject to this approach.

Key words: Educational Legislation, learning difficulties, educational evaluation,

\footnotetext{
1 Doctorante Universidad Arturo Prat del Estado de Chile, Chile. Dirección electrónica: nelly.ruiz@live.com.ar Orcid https://orcid.org/0000-0002-2794-3477
}

Ensayo recibido: 29 de octubre, 2019

Enviado a corrección: 28 de febrero, 2020

Aprobado: 20 de abril, 2020 


\section{Introducción}

El presente trabajo tiene como objetivo visibilizar y analizar, desde la perspectiva de la Legislación nacional, el marco contextual de la educación inclusiva en Colombia. Además, pretende describir la gestión de las Necesidades Educativas Especiales no Convencionales (NEE-NC) dentro de las instituciones de educación básica y media, en relación con los saberes de la población docente y directiva, pero también con respecto a la aplicación de garantías de igualdad de oportunidades y participación en instrumentos de evaluación institucional y nacional, desde la perspectiva del concepto actual de calidad.

En cuanto a los derechos humanos, el análisis debe conducir a dos extremos: el individual y el social, donde se puede situar la educación como una necesidad básica, común a todas las personas, referente a aspectos y objetivos de desarrollo humano e indicadores de crecimiento personal y social (Luque, 2009). La evidencia de grupos poblacionales que, en contextos de vulnerabilidad económica y social presentan una situación de desventaja, permite revelar respuestas del sistema educativo convencional que generan procesos de exclusión y discriminación, en virtud de un modelo homogéneo de educación.

El Ministerio de Educación Nacional (2012, p. 5) define algunas condiciones cuya apuesta por la inclusión debe ser considerada, como son:

... étnicas (Afrodescendientes, Rom y Raizales), con Necesidades Educativas Especiales (con discapacidad o con capacidades o talentos excepcionales), personas de talla baja (enanismo), jóvenes y adultos iletrados, afectadas por la violencia (en situación de desplazamiento, niños, niñas y jóvenes desvinculados de grupos armados al margen de la ley y adultos reinsertados), menores en riesgo social (niños, niñas y jóvenes trabajadores, adolescentes en conflicto con la ley, y niños y niñas en protección); habitantes de frontera y población rural dispersa.

Lo anterior supone que se han de generar acciones encaminadas a propiciar ambientes de enseñanza/aprendizaje, capaces de desarrollar las potencialidades académicas y personales de esta población. Sin embargo, no se evidencia en Colombia una línea de atención que muestre experiencias exitosas y acciones orientadoras respecto de la inclusión de personas con estas características.

La incorporación de los principios de la educación inclusiva a nivel mundial tiene como base la Declaración Universal de los Derechos Humanos (1948) que, en su artículo 26, decretó la educación como un derecho "que tiene por objeto el pleno desarrollo de la 
personalidad humana". Por su parte, la Convención sobre los Derechos del Niño (1989) garantiza la obligatoriedad de aplicación de los Derechos Humanos en la Niñez y la Infancia por parte de los Estados.

En la década de los años noventa toma curso un análisis particular con la Declaración Mundial sobre Educación para Todos y el Marco de Acción para satisfacer las Necesidades Básicas de Aprendizaje, aprobados por la Conferencia Mundial sobre Educación para Todos (1990), con lo cual, la Organización de las Naciones Unidas para la Educación, la Ciencia y la Cultura (UNESCO) estableció como objetivo generar un movimiento que trajera consigo una educación realmente inclusiva, de manera que todos los aportes apuntaran a concebir el mayor índice de cobertura posible y con ello, comprender la discapacidad como una oportunidad que conduciría a transitar nuevos caminos educativos y a construir y ofrecer nuevas herramientas para la enseñanza y el aprendizaje con miras al Nuevo Milenio.

La Academia fue convocada a producir documentos que solidificaran y soportaran los análisis de experiencias significativas, así como nuevas propuestas que atendieran las necesidades e inquietudes sobre la integración de las diferentes condiciones de aprendizaje a aulas regulares. Esto motivó a los países a continuar generando políticas públicas que darían lugar a unas concepciones innovadoras y consecuentes prácticas de inclusión.

Desde entonces, mediante la normatividad establecida en políticas públicas, los Estados han rediseñado sus objetivos de cara a las exigencias del siglo XXI, en procura de una educación para todos, compartida en Foros a nivel mundial como el que se llevaría a cabo en Dakar (2000), en el cual las personas en condición de discapacidad ocupan una de las primeras líneas de atención y rediseño al destacar que: El Marco de acción de Dakar (2000) es la confirmación de la visión formulada en Jomtien (1990) y que expresa el compromiso colectivo de la comunidad internacional de perseguir una estrategia amplia, con el objeto de garantizar que en el lapso de una generación se atiendan las necesidades básicas de aprendizaje de toda la población escolar y que esta situación se mantenga después.

La evaluación, definida en Colombia por el Ministerio de Educación Nacional (2017) como "una herramienta para promover el aprendizaje efectivo, la pertinencia de la enseñanza, la comprensión de las metas de aprendizaje y la motivación del estudiante" (p. 1), no parece tener una connotación de poder determinante dado que, por definición, parece resolver todo lo importante. Sin embargo, las diferentes condiciones y posibilidades de las personas en el ámbito escolar han dado lugar a tanta segregación que no solo se han 
generado los perjuicios para esta población, descritos antes, sino que se invisibilizaron condiciones no connotadas como convencionales (Rodríguez-Revelo, 2017).

Son pocos los estudios recientes que han evidenciado, en diferentes esferas, la situación de esta población escolar que se encuentra en la franja media, es decir, que no corresponde a la regularidad, ni tampoco a la discapacidad. Uno de ellos es el caso propuesto por Beltrán, Martínez y Vargas (2015), quienes al hacer un estudio comparativo entre las políticas de Estado en Colombia y las medidas adoptadas por España concluyen que, a pesar de la educación inclusiva, reglamentada en Colombia por diversas acciones normativas, continúa prevaleciendo el enfoque de discapacidades físicas y sensoriales, situación que evidencia una lenta incorporación de medidas efectivas para atender los demás grupos en riesgo de exclusión (NEE-NC).

Adicionalmente, Fragoso-Luzuriaga (2019) hace referencia a la importancia del desarrollo de la Inteligencia Emocional desde la dinámica relacional en los diferentes espacios sociales, como base de la regulación de las relaciones interpersonales, lo que se vería reflejado en los avances de la investigación, al contar con un buen clima en el caso de personas investigadoras.

Si bien es cierto, las personas investigadoras llegan a serlo luego de una amplia formación académica y disciplinar, es igualmente válido que el desarrollo emocional forma parte del desarrollo humano en las diferentes etapas por las que atraviesa y que en la niñez y en la adolescencia se consolida en buena medida. Es aquí donde, por diversos factores, se dan condiciones y directrices erráticas para la educación emocional y algunas personas no logran asertividad en sus relaciones interpersonales, por lo que devienen en el fracaso escolar al no ser vistas como estudiantes en condición especial de aprendizaje y no encontrarse inmersas en un sistema que contemple la educación emocional como parte fundamental del éxito académico.

Lo anterior revela que siguen presentes muchos elementos que determinan el fracaso escolar en estudiantes de la franja media, pues, si bien el sistema educativo contempla en algunas de sus políticas de Estado esta problemática, no interviene efectivamente para generar protocolos de atención en el caso de personas con estilos de aprendizaje no convencionales, traumatizadas por la violencia intrafamiliar, la pobreza, el conflicto armado, el desplazamiento, la migración, la adopción (cuando esta resulta desafortunada), las adicciones, algunos trastornos mentales de la amplia gama establecida por la Organización 
Mundial de la Salud (OMS), el retraso leve de origen desconocido, el estrés postraumático y otros.

A esta realidad excluyente que vive dicha población se añaden las políticas de estado que permiten, además, la aplicación de pruebas estandarizadas para medir la "calidad de la educación”. Dentro de esta línea de pensamiento, en Colombia, los Ministerios y las entidades encargadas de la definición de necesidades educativas especiales actualizaron la lista de categorías de discapacidad (Tabla 1), y aunque estas categorías son de amplio espectro, continúan fuera del radar otras situaciones, como las mencionadas en el párrafo anterior, que subyacen invisibles, de estudiantes cuyas condiciones especiales de aprendizaje no han sido contempladas.

La Legislación Educativa vigente en Colombia convierte a esta franja poblacional en un "obstáculo" para la conveniencia de las instituciones educativas de figurar en los ranking de calidad de la educación, ya que al ser invisible para el Sistema y no poder ser ubicada en las categorías definidas de discapacidad, es contemplada y atendida dentro de la regularidad, generando así unos resultados negativos para las instituciones educativas que evalúan a esta población atendiendo a los patrones de expectativa estatal, con lo que se obtiene como consecuencia la pérdida del año escolar o el retiro de la o el estudiante tras fallidos intentos de aprobación en un sistema para el que su particularidad permanece oculta.

Por lo anterior, se pretende visibilizar la situación de esta población con el fin de generar conciencia y compromiso en la aplicación de unos lineamientos de evaluación que, centrados de manera preeminente en el registro de avances, particularicen la situación de cada niña, niño o adolescente, desde sus singulares posibilidades de aprendizaje.

En suma, este Documento sintetiza una creciente inquietud fundamentada en la propia experiencia y en el análisis de la situación expuesta, la cual se convierte en un problema de investigación cuyo objetivo es aportar a la atención de esa franja poblacional y a la consecuente transformación de la política pública y de la práctica pedagógica, hacia una educación verdaderamente inclusiva.

En este sentido, el presente Trabajo hace un recorrido que inicia con la descripción de la situación actual de la problemática en Colombia, teniendo como base la realidad y la política pública, para proseguir con un análisis de la población perteneciente a la franja media y de sus necesidades no cubiertas por el sistema, así como de las actuales condiciones referidas al sistema docente y finaliza con la propuesta de unos lineamientos a 
tener en cuenta para dar inicio a la restitución de derechos de enseñanza y evaluación de la población con necesidades educativas no convencionales de aprendizaje.

\section{La experiencia colombiana}

Las políticas públicas más significativas, que rigen en Colombia, relacionadas con el objeto del presente estudio son las siguientes:

1. Declaración Universal de los Derechos Humanos 1948, art. 26.

2. Constitución Política de Colombia 1991, arts. 13, 47 y 67 (Const, 1991.)

3. Ley 115 de 1994 (arts. 46 y 47). (Congreso de la República de Colombia, 1994)

4. Ley Estatutaria 1618 (2013, febrero 27). Por la cual se establecen las disposiciones para garantizar el pleno ejercicio de los derechos de las personas con discapacidad. (Bogotá: Ministerio de Salud).

5. Ley 1346 (2009, julio 31. Por la cual se aprueba la "Convención sobre los Derechos de las personas con discapacidad", adoptada por la Asamblea General de la Naciones Unidas el 13 de diciembre de 2006.

6. Decreto 1421 de 2017. (Ministerio de Educación Nacional):

Por el cual se reglamenta, en el marco de la educación inclusiva, la atención educativa a la población con discapacidad. La discapacidad es definida en este Decreto en el artículo 9, como sigue: "Estudiante con discapacidad: persona vinculada al sistema educativo en constante desarrollo y transformación, con limitaciones en los aspectos físico, mental, intelectual o sensorial que, al interactuar con diversas barreras (actitudinales, derivadas de falsas creencias, por desconocimiento, institucionales, de infraestructura, entre otras), pueden impedir su aprendizaje y participación plena y efectiva en la sociedad, atendiendo a los principios de equidad de oportunidades e igualdad de condiciones.

7. Guía Construyendo capacidad institucional para la atención a la Diversidad (Ministerio de Educación Nacional, 2012)

8. Ley 1098 (2006, agosto 11). Congreso de la República de Colombia.

9. Guía Lineamientos Generales para la atención educativa a población vulnerable y víctima del conflicto (Ministerio de Educación Nacional, 2014) 
Aunque se busca definir la discapacidad en los documentos citados, son tantas y tan variadas las condiciones de los estudiantes, que fueron establecidas unas categorías de discapacidad que con el tiempo se han ido modificando.

En la Tabla 1 se puede visualizar la última actualización que se hizo en el año 2017 con la participación de los Ministerios de Salud y Educación.

Tabla 1

Definición de categorías de discapacidad según el Ministerio de Educación Nacional y el Ministerio de Salud de Colombia

\begin{tabular}{|c|c|c|}
\hline \multicolumn{3}{|c|}{ DEFINICIONES DE CATEGORÍAS DE DISCAPACIDAD } \\
\hline 1 & Movilidad & $\begin{array}{l}\text { Algunos subtipos de este tipo de discapacidad son: } \\
\text { - Parálisis Cerebral sin compromiso cognitivo. } \\
\text { - Parálisis: cuadriplejia (cuatro miembros), hemiplejia (medio lado } \\
\text { derecho o izquierdo), monoplejía (un solo miembro), paraplejia } \\
\text { (dos miembros superiores o inferiores). } \\
\text { - Distrofia muscular. } \\
\text { - Osteogénesis imperfecta (niños con huesos de cristal). } \\
\text { - Lesión neuromuscular. } \\
\text { - Espina bífida. } \\
\text { - Otras relacionadas. }\end{array}$ \\
\hline 2 & Sensorial auditiva & $\begin{array}{l}\text { En esta categoría se reportan las personas-estudiantes que } \\
\text { presentan, en forma permanente, alteraciones en las funciones } \\
\text { auditivas, como localización, tono, volumen y calidad de los sonidos. }\end{array}$ \\
\hline 3 & Sensorial visual & $\begin{array}{l}\text { En esta categoría se reportan los estudiantes que presentan, en } \\
\text { forma permanente, alteraciones para percibir la luz, forma, tamaño, o } \\
\text { color. Se deben reportar estudiantes con baja visión y ceguera. }\end{array}$ \\
\hline 4 & Sensorial voz y habla & $\begin{array}{l}\text { En esta categoría se encuentran aquellas personas que presentan, } \\
\text { en forma permanente, alteraciones en el tono de la voz, la } \\
\text { vocalización, la producción de sonidos y la velocidad del habla. } \\
\text { También, las personas con dificultades graves o importantes para } \\
\text { articular palabras (mudez, tartamudez), es decir, con alteraciones } \\
\text { graves del lenguaje. }\end{array}$ \\
\hline 5 & Sordoceguera & $\begin{array}{l}\text { Es una discapacidad que resulta de la combinación de dos } \\
\text { deficiencias sensoriales (visual y auditiva), y que genera en quienes } \\
\text { la presentan problemas de comunicación únicos y necesidades } \\
\text { especiales derivadas de la dificultad para percibir el entorno. }\end{array}$ \\
\hline 6 & $\begin{array}{l}\text { Discapacidad } \\
\text { intelectual }\end{array}$ & $\begin{array}{l}\text { En esta categoría se encuentran aquellas personas-estudiantes que, } \\
\text { en forma permanente, presentan alteraciones en las funciones } \\
\text { intelectuales y cognitivas. Se refiere a aquellas personas que podrían } \\
\text { presentar, en el desarrollo de sus actividades cotidianas, diferentes } \\
\text { grados de dificultad en la adquisición y aplicación de los elementos } \\
\text { del aprendizaje para la ejecución de actividades de cuidado personal, } \\
\text { del hogar, comunitarias, y sociales entre otras, así como dificultades } \\
\text { para interactuar con otros de una manera apropiada dentro de su } \\
\text { entorno social. Para lograr una mayor independencia se requiere de } \\
\text { apoyos especializados, terapéuticos y pedagógicos; entre estos } \\
\text { casos están: } \\
\text { - Síndrome de Down. } \\
\text { - Otros síndromes con compromiso intelectual. } \\
\text { - Trastorno cognitivo. }\end{array}$ \\
\hline
\end{tabular}




\begin{tabular}{|l|l|l|}
\hline \multicolumn{2}{|c|}{ DEFINICIONES DE CATEGORÍAS DE DISCAPACIDAD } \\
\hline $\mathbf{7}$ & & $\begin{array}{l}\text { En esta categoría se encuentran aquellas personas-estudiantes que } \\
\text { presentan, en forma permanente, alteraciones de conciencia, } \\
\text { orientación, energía, impulso, atención, temperamento, memoria, } \\
\text { personalidad y en las conductas psicosociales, entre otras. Personas } \\
\text { con desórdenes mentales expresados en comportamientos o } \\
\text { expresiones emocionales inadecuadas como la esquizofrenia, la } \\
\text { bipolaridad, trastornos de ansiedad, manifestados a través de } \\
\text { trastorno obsesivo compulsivo, mutismo selectivo, psicosis, entre } \\
\text { otras, quienes requieren de tratamiento psiquiátrico para poder ser } \\
\text { incluidos en el sistema educativo, ya que pierden el contacto con la } \\
\text { realidad. }\end{array}$ \\
\hline $\mathbf{8}$ & $\begin{array}{l}\text { piscapacidad mental } \\
\text { psicosocial } \\
\text { espectro autista }\end{array}$ & $\begin{array}{l}\text { Afecta el desempeño de funciones intelectuales, psicosociales, la voz } \\
\text { y el habla, incidiendo en las relaciones interpersonales. }\end{array}$ \\
\hline $\mathbf{9}$ & $\begin{array}{l}\text { Discapacidad } \\
\text { sistémica }\end{array}$ & $\begin{array}{l}\text { Cuando las condiciones de salud ocasionan limitaciones en la } \\
\text { actividad y restricciones en la participación debido a enfermedades } \\
\text { relacionadas con los sistemas cardiovascular, hematológico, } \\
\text { inmunológico, respiratorio, del sistema digestivo, metabólico, } \\
\text { endocrino, de la piel, enfermedades terminales, entre otras. }\end{array}$ \\
\hline $\mathbf{1 0}$ & Múltiple discapacidad & \begin{tabular}{l} 
Cuando tiene 2 o más discapacidades. \\
\hline
\end{tabular}
\end{tabular}

Fuente: Elaboración propia, con información del Ministerio de Educación Nacional de Colombia, 2017.

\section{Las necesidades educativas especiales no convencionales en Colombia: retos y prospectivas}

La categorización citada anteriormente, así como la normatividad legal vigente permiten un reconocimiento a los esfuerzos del Estado representado en sus legisladores y en los Ministerios de Educación Nacional y de Salud, por contemplar todas aquellas condiciones que requieren el tránsito hacia una educación inclusiva. Sin embargo, aunque la descripción de las dificultades no convencionales de aprendizaje y de los canales preferentes o de los estilos de aprendizaje se hallan consignados en algunos apartes de la legislación vigente, no se encuentran reflejados en la práctica escolar.

Los mayores desafíos de la educación totalmente inclusiva en Colombia abarcan la existencia de barreras actitudinales, la baja participación de los docentes, la baja eficiencia en la gestión de recursos, el rediseño de los programas de formación docente y la resistencia al cambio de paradigma de una escuela homogenizada a una escuela tan plural como la población escolar que la integra. (Ministerio de Educación Nacional, 2012, p. 5)

Igualmente, algunos autores han considerado que la tarea no está acabada y que algunas personas podrían encontrarse fuera de esta categorización, como Florido (2018), quien afirma que: 
La correlación entre la condición de discapacidad y la vulneración del derecho a la educación sigue siendo relevante, y que esta situación se presenta por el concierto de diversos actores (estudiantes, docentes, sociedad) que, en las circunstancias propiciadas por la estandarización de la educación, hacen que sea muy difícil combatir la segregación. (p. 26)

Al mencionar la dificultad de establecer criterios universales de inclusión por la imposibilidad de sincronizar a todos los actores desde las diversas aristas del escenario de aprendizaje, Florido ofrece varios elementos de análisis indicando que, las personas relacionadas con el campo educativo son tan diversas como las niñas, los niños y la población adolescente en términos de sus características, sus posibilidades, sus contextos y sus estilos de aprendizaje; esto explicaría la mortalidad académica, la deserción escolar o el bajo rendimiento, los cuales se encuentran a la vista de cualquier observador.

Lo anterior nos lleva a pensar en lo improductivo que puede resultar, en algunos casos, un proceso tan complejo como es la educación. Andrés y Martínez-Olmo (2008) lo confirman cuando concluyen: "El conocimiento en el siglo XXI se caracteriza por su crecimiento vertiginoso, su continua fragmentación y su rápida obsolescencia" (p. 10). Esto supone que, según como incorporemos la educación a nuestras experiencias, puede hacer del conocimiento una herramienta potencialmente inútil para luchar contra las crecientes demandas de la realidad social. Para el tema que nos ocupa, uno de los factores de complejidad social podría ser la multidireccionalidad de la educación en el contexto vocacional. La diversidad de las profesiones, artes y oficios que demanda la sociedad actual está lejos de ser satisfecha desde la estrecha óptica de los estándares de calidad y desde los limitados currículos que minan posibilidades tan variadas como las que ya contemplaba, hace más de dos décadas, Gardner (1997) al definir la inteligencia como:

La capacidad de resolver problemas o de elaborar productos que sean valiosos en uno o más ambientes culturales. Se trata de una definición que nada dice acerca de las fuentes de tales capacidades o de los medios adecuados para «medirlas». (p. 10)

Así, al someter a una persona a una evaluación cuyo resultado determinará el avance o el estancamiento académico cuando sus características, estilos, posibilidades o capacidades de aprendizaje no se pueden enmarcar en la regularidad ni en la discapacidad, estamos generando temor y tensión en esa parte que debería fluir sin limitaciones, debiendo 
ser la más placentera del proceso, al ser la que permite a cualquier aprendiz, exponer sus competencias.

Desde la realidad actual de las diversas respuestas de aprendizaje, algunos científicos y algunas científicas han seguido adentrándose en la complejidad del cerebro, como la Doctora Julia Harper (2019) quien fue invitada a nuestro país con motivo de la VI Cumbre de Líderes por la Educación para compartir su experiencia con el tratamiento de las dificultades de aprendizaje no convencionales en su Centro de Atención Therapeed y y quien afirma que:

Estas dificultades surgen porque el cerebro no funciona bien. Es decir, cuando no puede recibir información y no puede entenderla ni procesarla adecuadamente. Eso afecta la coordinación motora, el pensamiento, la relación con los demás. Hay varias explicaciones para entender este fenómeno. La primera es obvia: cuando la persona tiene un daño físico en la estructura del cerebro, cuando sus receptores no funcionan o se dañan. Estas son personas ciegas o sordas. La segunda es cuando tienen un problema genético, síndrome de Down, por ejemplo. La tercera es más sutil: cuando todo en apariencia funciona bien en el cerebro, pueden oír y ver, pero sus conexiones están en cortocircuito. El problema en ese caso es cómo el cerebro se comunica y emite órdenes. Nosotros nos enfocamos en este último y utilizamos la ciencia de neuro plasticidad para ayudar a que las personas aprendan a usar otra parte del cerebro que no tiene daños y compense la parte que no funciona bien. Usamos la neuro plasticidad para reparar las conexiones dentro del cerebro. (párr.2)

Con las afirmaciones de la Dra. Harper se confirma que, además de las dificultades obvias por una discapacidad, existen otras dificultades sutiles que nadie notaría a simple vista, nadie, ni las políticas de Estado.

\section{El papel del docente en el escenario de inclusión}

Estudios recientes han tocado temas tan sensibles para la inclusión, como el cuestionamiento a la preparación que las personas que eligen la docencia deberían tener para poder reconocer esas alteraciones del aprendizaje que no son tan visibles como las discapacidades contempladas en la Tabla 1. Guzmán (2018) considera que:

La educación inclusiva es uno de los retos que supera las barreras del tiempo y permite que los actores educativos se involucren en cada práctica para enriquecer los ambientes de aprendizaje. Por ello, la calidad humana que brinda el personal docente 
responde a la empatía que comunica en el quehacer pedagógico. Consiguientemente, revela la concepción humanista para colocarse en el lugar del otro y al participar de las actividades colectivas favorecer las heterogeneidades presentes en la educación inclusiva. (p. 3)

Evidentemente, se trata de un ideal que conduciría, en un alto porcentaje, a detectar las particularidades invisibles de un sistema rígido que atiende estándares de calidad, pero que olvida sus contextos y a los seres humanos singulares y complejos que contiene. En la misma línea de análisis sobre la formación docente Castillo-Briceño (2015) afirma que:

Para las unidades de desarrollo docente la educación inclusiva constituye una oportunidad para hacer la tarea educativa de manera diferente. Para ello es necesario articular teoría y práctica, tener conciencia de las teorías que se aplican, propiciar espacios reflexivos para ampliar la percepción de lo que significa educar en y para la diversidad. Por lo tanto, avanzar hacia una educación inclusiva requiere partir de la realidad del entorno, tener claro hacia donde se orienta la educación e implementar ambientes adecuados. (pp. 28-29)

No se trata solamente de tener con las(los) niñas(os) y las(los) adolescentes una comunicación asertiva que le brinde a los docentes la posibilidad de derribar los miedos de la educación tradicional, ni de permitirle a la niñez a su cargo expresar sus necesidades de flexibilización y de adaptación curricular para alcanzar el éxito académico, sino que se trata del concierto de toda la sociedad y de la formación integral del docente; que le permita afinar la mirada para captar las demandas de toda la población en su afán por ser incluida en un sistema que, al marcar la discapacidad, invisibiliza a esta franja media sumiéndola en el abandono de sus posibilidades de consolidación personal y académica.

La óptima preparación docente para la inclusión y que está contemplada en algunas políticas de Estado debería ser parte de la formación profesional. El Decreto 1421 de 2017 (art. 8) reza:

Incluir en el Plan Territorial la formación docente en aspectos básicos para la atención educativa a estudiantes con discapacidad de conformidad con lo previsto en la presente sección y, fortalecer este tema en los procesos de inducción y reinducción de los docentes y directivos docentes. 
Ninguna persona debería ejercer la docencia sin saber todo lo necesario para una auténtica educación inclusiva, pero este requisito no se ha consolidado en las Facultades de Educación ni tampoco se revisa en el contexto educativo. Es evidente el afán del Estado por mostrar la normatividad, pero de nada sirve si esta no es objeto de control y vigilancia. De acuerdo con lo analizado en las necesidades de inclusión total, el personal docente debería salir de las Facultades de Educación preparado, entre otras cosas, para:

a. Comprender la diferencia entre la flexibilización y la adaptación curricular, de conformidad con la discapacidad o las dificultades no convencionales de aprendizaje.

b. Generar una comunicación asertiva que permita a los escolares a su cargo expresar sin temores sus condiciones de aprendizaje en un proceso que facilite sus logros académicos y emocionales.

c. Detectar las manifestaciones de episodios emocionales que evidencien desequilibrios mentales y dar una asertiva intervención primaria que permita, con el apoyo familiar, el direccionamiento del niño, niña o adolescente hacia los servicios sanitarios acreditados para la atención que se requiere.

d. Conocer e inquietarse por identificar los factores de riesgo que afectan, actualmente, el equilibrio físico, social y emocional de la población escolar.

e. Tener conciencia de los diferentes tipos de inteligencia, estilos de aprendizaje, discapacidades, dificultades no convencionales de aprendizaje y demás condiciones particulares de la niñez.

f. Tener claridad sobre el uso y el sentido de las diferentes herramientas didácticas e incorporar, de manera creciente, todo tipo de recursos que posibiliten la visualización de las condiciones de aprendizaje individuales.

g. Reconocer el valor del trabajo en equipo y abrir las aulas a los servicios de apoyo terapéutico requeridos en el proceso de algunas(os) niñas(os) y adolescentes.

h. Trabajar en la implementación de asignaturas como música, teatro, origami, ajedrez, etc., las cuales contribuyen con el desarrollo cerebral y con el fortalecimiento de habilidades necesarias para la consolidación física, emocional, social e intelectual.

i. Dar relevancia al ejercicio físico y mental, ya que un cuerpo y un cerebro que no se ejercitan tienden al deterioro de sus facultades intrínsecas. 
Aunque todo lo anterior podría generarse en formación posgradual se hace necesario que sea de carácter obligatorio en el pregrado, ya que estar preparado para la inclusión no debe ser una opción, es una necesidad creciente de la sociedad actual. De igual manera está en aumento la necesidad de contar con maestros cuidados emocionalmente para que puedan ser cuidadores aptos de la población escolar a su cargo (García-Barrera, 2017). En este aspecto son de resaltar los invaluables aportes de Naranjo (2007, párr. 3) cuando afirma:

Educar a los educadores no se refiere a la formación en conocimientos técnicos como las matemáticas o las lenguas, sino a aspectos más profundos. Para ello propone que los profesores se adentren en el mundo del desarrollo personal. Que vivan su propio proceso de transformación, aunque enseñen asignaturas de ciencias puras. Es difícil ayudar a alguien a aceptar el error, a superar sus conflictos o a no etiquetar a la primera de cambio si no trabaja primero sus propias dificultades.

Con lo anterior queda evidente que el Estado debe revisar sus políticas partiendo de la claridad de los conceptos y de un cambio de paradigma sobre lo que significa la calidad educativa. Resulta perjudicial establecer patrones de referencia universales sobre una población tan compleja y diversamente hábil (personas que presentan diferencias físicas, cognitivas o sensoriales, pero que tienen habilidades y capacidades funcionales que les permiten expresar y realizar de manera diferente y con dignidad su proyecto de vida) desde la discapacidad hasta las altas capacidades y talentos.

Al respecto y con el ánimo de compartir una condición diferente que sufre casi las mismas consecuencias por falta de políticas de Estado claras y verdaderamente inclusivas, Covarrubias-Pizarro (2018, p. 66) se inquietó por el avance del concepto de aptitudes sobresalientes al de altas capacidades y talentos. En su estudio plantea:

Ofrecer el panorama y el análisis de lo que implica el término de altas capacidades remite a una comprensión de la población que se encuentra por arriba del promedio y a que en el quehacer cotidiano se puedan identificar mayores opciones para su detección oportuna y adecuada intervención. Las implicaciones que trae consigo el uso de la conceptualización actual pueden repercutir mientras se sigan ofreciendo alternativas limitadas de intervención para el desarrollo de las capacidades de las(os) niñas(os) y de los jóvenes sobresalientes y mientras las(los) mantengan al margen de nuevas estrategias. 
En este caso, se trata de otra población invisibilizada por falta de claridad en cuanto a su categorización, pero cuyas implicaciones son las mismas con las que deben lidiar la niñez y la adolescencia correspondientes a la franja media, objeto de este estudio.

La normatividad parece contemplarlo todo, pero en los escenarios educativos, en su cotidianidad, los actores se preguntan ¿Cómo?... ¿Cómo hacer un Plan Individualizado de Ajustes Razonables (PIAR) sin haber hecho un diagnóstico exhaustivo de cada estudiante? ¿Cómo reconocer en la «pereza» una forma de evasión, cuando se debe lidiar con una situación de violencia o abuso intrafamiliar? ¿Cómo se hace para dar a cada quien lo que le corresponde sin motivación ni herramientas? Nada más atinado que lo expuesto por Echeita (2017) cuando afirma:

La contradicción flagrante entre lo que se dice en las normas y lo que realmente ocurre en muchos centros educativos, "entre lo dicho y lo hecho", genera enormes tensiones y desgarros emocionales que afectan muy negativamente a muchos estudiantes vulnerables y a sus familias. (p. 17)

\section{Proposición}

Parte del conjunto de lineamientos que consolidarían el principio de atención de la problemática tratada debe focalizarse en el registro de avances de cada estudiante, sin patrones de referencia. Esta acción busca liberar a la educación del poder que la tradición ha depositado en la evaluación para determinar el futuro de cada persona. Un poder tan enorme como la decisión de seguir vivo, que algunas personas han tomado ante el fracaso académico, y que en la mayoría de los casos lo relacionan con una pérdida de sentido de la propia existencia.

Se sugiere hacer un diagnóstico de cada estudiante, que retome y amplíe las conductas de entrada hasta ahora asimiladas solamente a los prerrequisitos relacionados con las habilidades académicas que cada persona debe tener al ingresar a un grado superior. Ampliar la mirada implica una serie de actividades desarrolladas por un equipo interdisciplinario que permita identificar en cada estudiante su contexto, su historial académico, sus estilos de aprendizaje, sus canales de percepción (visual, auditivo, kinestésico), sus gustos, sus talentos, su nivel de lectura y escritura, sus pasatiempos favoritos, sus principales inclinaciones en el uso del tiempo libre, sus antecedentes médicos, sus antecedentes históricos y sociales, su desarrollo psicomotriz y del lenguaje. No podemos tratar de educar a una criatura desconociendo su potencial, su historia y su contexto. 
El registro de antecedentes, características y posibilidades debe corresponder a un primer período exploratorio que permita identificar la estrategia más adecuada en cada caso, tiempo en el cual se podrán identificar grupos pares en sus inclinaciones académicas, artísticas, deportivas y culturales, con lo cual se posibilita establecer grupos de estudio, de cultura física, de exploración científica, artística y cultural, de lectura y producción escrita.

Sin más pautas de referencia que el propio nivel de avance, cada persona podría estar acompañada en su exploración del mundo y direccionada a abrir las puertas de la ciencia, las humanidades, la tecnología, el deporte, el arte, la cultura o el sinnúmero de posibilidades que su realización y su futuro demanden.

Lo anterior se aleja de sus posibilidades si continúa la incoherencia del Estado de exigir, por un lado, la inclusión total y por otro, la medición de los estándares de calidad educativa en dos niveles: la regularidad y la discapacidad. La verdadera calidad de las instituciones debería medirse por la calidad de vida de sus egresados, por lo felices que sean, por el bien que aporten a la humanidad en todas las esferas elegidas de formación y, por supuesto, en los ámbitos personal y social.

\section{Conclusiones}

A partir de lo expuesto se evidencia una discordancia entre la planificación, la comunicación y la ejecución del currículo de acuerdo con los lineamientos establecidos por la legislación vigente en Colombia. Esta situación supone limitaciones para el acceso a la educación inclusiva de población con NEE-NC (Necesidades Educativas Especiales no Convencionales).

En particular, la evaluación en la forma en que la conocemos ha dotado de un poder desmedido a múltiples actores e intenciones, y en algunos casos ha interferido en el deseo de aprender, ha invisibilizado inteligencias que no contempla, ha pretendido que los seres humanos puedan ser ubicados dentro de un patrón, desconociendo su enorme complejidad y ha equivocado el concepto de calidad educativa. Las políticas de Estado no cubren la necesidad de una educación totalmente inclusiva por la falta de claridad conceptual, de inspección sobre la formación docente, de herramientas y de condiciones de control y vigilancia.

Hoy, todavía, existen muchos seres humanos anulados por los efectos nocivos de la evaluación que conocemos y hay muchos más que no respiran en este mundo por la misma situación. El sentido de la educación podría peligrar en manos de uno de sus componentes 
más empoderados socialmente: la evaluación. Se hace necesaria una reflexión sobre el enorme poder que la historia le ha conferido y sus efectos tan demoledores para la población estudiantil que ocupa la franja media descrita en el presente Documento.

\section{Referencias}

Andrés, Joan Mateoy Martínez-Olmo, Francesc. (2008). La evaluación alternativa de los aprendizajes. Barcelona, España: Octaedro.

Beltrán, Yolima Ivonne; Martínez, Yexica Lizethy Vargas, Ángela. Sofía. (2015). El sistema educativo colombiano en el camino hacia la inclusión: Avances y retos. Educación y Educadores, 18(1), 62-75. doi:10.5294/edu.2015.18.1.4

Castillo-Briceño, Cristina. (2015). La educación inclusiva y lineamientos prospectivos de la formación docente: una visión de futuro. Revista Electrónica Actualidades Investigativas e Educación, 15(2), 1-33. Doi http://dx.doi.org/10.15517/aie.v15i2.18534

Colombia. (04 de julio, 1991). Constitución Política. Recuperado de http://wsp.presidencia.gov.co/Normativa/Documents/Constitucion-Politica-Colombia.pdf

Congreso de la República. (31 de Julio, 2009). Ley 1346. Por medio de la cual se aprueba la "Convención sobre los Derechos de las personas con discapacidad". Recuperado de http://www.secretariasenado.gov.co/senado/basedoc/ley 1346 2009.html

Congreso de la República de Colombia. (8 de Febrero, 1994). Ley 115 . Ley General de Educación. Bogotá, Colombia. Recuperado de Ministerio de Educación Nacional: https://www.mineducacion.gov.co/1621/articles-85906 archivo pdf.pdf

Congreso de la República de Colombia. (08 de noviembre, 2006). Ley 1098 de 2006 Código de Infancia y Adolescencia. Bogotá, Colombia. Recuperado de http://www.secretariasenado.gov.co/senado/basedoc/ley 1098 2006.html

Constitución Política de Colombia. (1991). Recuperado de https://pdba.georgetown.edu/Constitutions/Colombia/colombia91.pdf

Covarrubias-Pizarro, Pedro. (2018). Del concepto de aptitudes sobresalientes al de altas capacidades. Rev. investig. educ, 9(17), 53-67. Recuperado de http://www.scielo.org.mx/scielo.php?script=sci arttext\&pid=S2448$85502018000200053 \& \operatorname{lng}=\mathrm{es} \& \ln \mathrm{n}=\mathrm{es}$

Declaración universal de los Derechos Humanos. (1948). Recuperado de https://www.ohchr.org/EN/UDHR/Documents/UDHR Translations/spn.pdf

Echeita Sarrionandía, Gerardo. (2017). Educación inclusiva. Sonrisas y lágrimas. Aula Abierta, 46, 17-24. doi: https://doi.org/10.17811/rifie.46.2017.17-24 
Florido Mosquera, Hugo Edilberto. (2018). Lineamientos curriculares para la incorporación de la Educación inclusiva en colegios públicos de Bogotá. Bogotá. Recuperado de https://repository.usta.edu.co/handle/11634/14892

Fragoso-Luzuriaga, Rocío. (2019). Importancia del desarrollo de la inteligencia emocional en la formación de personas investigadoras. Actualidades Investigativas en Educación, 19(1), 1-23. doi:DOI 10.15517/aie.v19i1.35410

García-Barrera, Alba. (2017). Las necesidades educativas especiales: Un lastre conceptual para la inclusión educativa en España. Ensaio: Avaliação e Políticas Públicas em Educação 25(96), 721-742.

Gardner, Howard. (1997). Estructuras de la mente. La teoría de las inteligencias múltiples. Bogotá, Colombia: Fondo de cultura económica.

Guzmán Huayamave, Kennya. (2018). La comunicación empática desde la perspectiva de la educación inclusiva. Actualidades Investigativas en Educación, 18(3) 1-18. doi:10.15517/AIE.V18I3.34211

Harper, Julia. (septiembre 19 de 2019). Los comportamientos de los padres afectan el cerebro de sus hijos (J. M. Bonilla, Ed.). El Espectador. Recuperado de https://www.elespectador.com/noticias/educacion/los-comportamientos-de-los-padresafectan-el-cerebro-de-sus-hijos-articulo-881931

Luque Parra, Diego Jesús. (2009). Las necesidades educativas especiales como necesidades básicas. Una reflexión sobre la inclusión educativa. Revista Latinoamericana de Estudios Educativos, 39(3 y 4) 201-223. Recuperado de http://repositoriocdpd.net:8080/handle/123456789/329

Ministerio de Educación Nacional. (2012). Educación inclusiva con calidad. Construyendo capacidad institucional para la atención a la Diversidad. Guía y Herrramienta. Bogotá. Recuperado https://www.researchgate.net/publication/316701329 Educacion inclusiva con calidad Construyendo capacidad institucional para la atencion a la diversidad Guia

Ministerio de Educación Nacional. (2014). Guía Lineamientos Generales para la atención educativa a población vulnerable y víctima del conflicto. Colombia: RET Fundación para el Fondo de Educación del Refugiado. Recuperado de http://redes.colombiaaprende.edu.co/ntg/men/archivos/LINEAMIENTOS-ATENCIONEDUCATIVA-EDICION-01-ENE2015-VERSION-ELECTRONICA.pdf

Ministerio de Educación Nacional. (2017). Manual de Educación privada: Evaluación. Recuperado de https://www.mineducacion.gov.co/1759/w3-article-179264.html

Ministerio de Educación Nacional. (agosto de 2017). Ley 1421. Por el cual se reglamenta en el marco de la Educación Inclusiva la atención educativa a la población con discapacidad.

Recuperado

de http://es.presidencia.gov.co/normativa/normativa/DECRETO\%201421\%20DEL\%2029\% 20DE\%20AGOSTO\%20DE\%202017.pdf 
Ministerio de Educación Nacional. (2017). Necesidades educativas especiales. Ajustes a las categorías de discapacidad, capacidades y talentos excepcionales. Recuperado de https://www.mineducacion.gov.co/1621/w3-printer-351622.html

Ministerio de Salud. (febrero 27 de 2013). Ley estatutaria 1618. Por medio de la cual se establecen las disposiciones para garantizar el pleno ejercicio de los derechos de las personas con discapacidad. Recuperado de http://www.secretariasenado.gov.co/senado/basedoc/ley 1618 2013.html

Naranjo, Claudio. (julio 8 de 2007). Cambiar la educación para cambiar el mundo (P. Jericó, Ed.) Madrid: Cuarto propio. Recuperado de https://elpais.com/elpais/2019/07/07/laboratorio de felicidad/1562533562 516197.html

Organización de las Naciones Unidas-ONU. (1948). Declaración Universal de los Derechos Humanos. París, Francia. Recuperado de https://www.un.org/es/documents/udhr/UDHR booklet SP web.pdf

Organización de las Naciones Unidas-ONU. (1989). Convención sobre los Derechos del Niño. Recuperado de https://www.un.org/es/events/childrenday/pdf/derechos.pdf

Organización de las Naciones Unidas para la Educación, la Ciencia y la Cultura (Unesco). (1990). Declaración mundial sobre educación para todos y marco de acción para satisfacer las necesidades básicas de aprendizaje. Jomtiem. Tailandia: Unesco.

Organización de las Naciones Unidas para la Educación, la Ciencia y la Cultura. (2007). Educación de calidad para todos: un asunto de derechos humanos. Buenos Aires, Argentina. Recuperado de https://unesdoc.unesco.org/ark:/48223/pf0000150272

Organización de las Naciones Unidas para la Educación, la Ciencia y la Cultura (Unesco). (1990). Declaración Mundial sobre educación para todos. Jomtien. Recuperado de https://www.oei.es/historico/efa2000jomtien.htm

Organización de las Naciones Unidas para la Educación, la Ciencia y la Cultura (Unesco). (1994). Declaración de Salamanca y Marco de Acción sobre Necesidades Educativas Especiales. Salamanca. Recuperado de https://unesdoc.unesco.org/ark:/48223/pf0000098427 spa

Organización de las Naciones Unidas para la Educación, la Ciencia y la Cultura (Unesco). (2000). Marco de Acción de Dakar: Educación para Todos: cumplir nuestros compromisos comunes (con los seis marcos de acción regionales). Dakar, Senegal. Recuperado de https://unesdoc.unesco.org/ark:/48223/pf0000121147 spa

Peppler-Barry, Ulrike. y Fiske, Edward. (2000). Foro mundial sobre la educación. Dakar. Senegal: Organización de las Naciones Unidas para la Educación, la Ciencia y la Cultura (Unesco). Recuperado de https://unesdoc.unesco.org/ark:/48223/pf0000121117 spa

Rodríguez-Revelo, Elsy. (2017). La estandarización en el currículo educativo: la punta del iceberg de la homogeneización. ALTERIDAD Revista de Educación, 12(2), 248-258. doi: https://dx.doi.org/10.17163/alt.v12n2.2017.10 
Revista indizada en

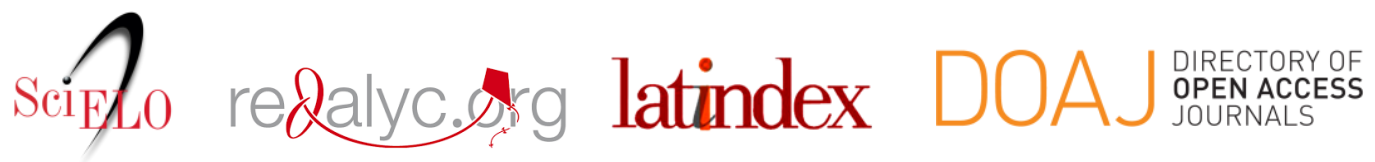

Distribuida en las bases de datos:

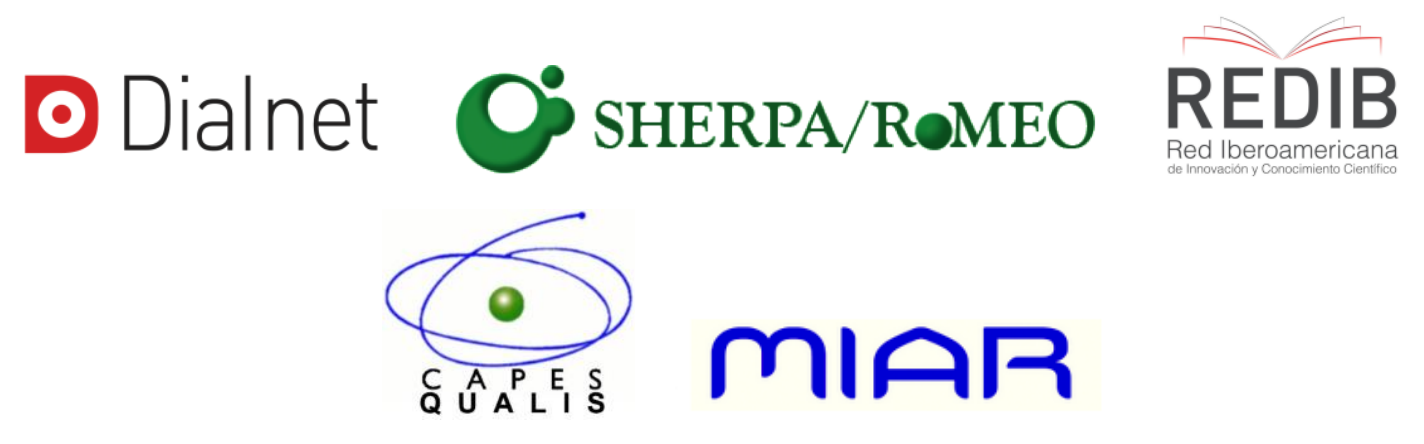

